\title{
Erratum: Effective Interaction between Active Colloids and Fluid Interfaces Induced by Marangoni Flows [Phys. Rev. Lett. 116, 078301 (2016)]
}

Alvaro Domínguez, P. Malgaretti, M. N. Popescu, and S. Dietrich

(Received 18 July 2016; published 11 August 2016)

DOI: 10.1103/PhysRevLett.117.079902

Recently, our attention was called upon Ref. [1], in which the issue of a heated solid particle near a fluid-gas interface was addressed theoretically and the velocity of the particle was computed. The formal similarity with our results lends support to our expressed view that temperature gradients induced by the particle can lead to the same phenomenology as concentration gradients do.

[1] A. M. Leshansky, A. A. Golovin, and A. Nir, Phys. Fluids 9, 2818 (1997). 